\title{
Erratum to: Hypo-pigmenting effect of sesquiterpenes from Inula britannica in $\mathrm{B}_{16}$ melanoma cells
}

\author{
Soo-Jin Choo • In-Ja Ryoo • Kwan Chul Kim • \\ Minkyun Na $\cdot$ Jae-Hyuk Jang $\cdot$ Jong Seog Ahn • \\ Ick-Dong Yoo
}

Published online: 25 March 2014

(c) The Pharmaceutical Society of Korea 2014

Erratum to: Arch. Pharm. Res.

$$
\text { 10.1007/s12272-013-0302-4 }
$$

Unfortunately, the authors, Kwan Chul Kim and Minkyun $\mathrm{Na}$ were omitted in the original version of the article.
The missing authors' names with affiliations and the correct order of the authors are updated in the article. In addition, the first author now has one more affiliation.

The online version of the original article can be found under doi:10.1007/s12272-013-0302-4.

S.-J. Choo - I.-J. Ryoo · J.-H. Jang · J. S. Ahn · I.-D. Yoo ( $ه)$ Chemical Biology Research Center, Korea Research Institute of Bioscience and Biotechnology, 30 Yeongudanji-ro,

Ochang, Cheongwon, Chungbuk 363-883, Republic of Korea e-mail: idyoo@kribb.re.kr

S.-J. Choo · M. Na

College of Pharmacy, Chungnam National University, 99

Daehak-ro, Yuseong-gu, Daejeon 305-764, Republic of Korea

K. C. Kim

Innoskin Co., Ltd., 209 BVC, Korea Research Institute of Bioscience and Biotechnology, 125 Gwahak-ro, Yuseong-gu, Daejeon 305-806, Republic of Korea 